\title{
Isomeric Folate-Conjugated Polymeric Micelles Bind to Folate Receptors and Display Anticancer Effects
}

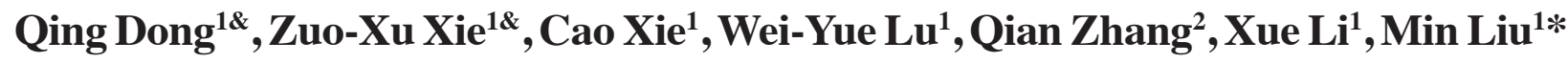

\begin{abstract}
The present study aimed to prepare and evaluate polymeric micelles conjugated with folic acid through $\alpha$ - or $\gamma$-carboxyl groups for antitumor efficacy. The isomeric block copolymers, $\alpha$ - and $\gamma$-folate-polyethyleneglycoldistearoyl phosphatidylethanolamine ( $\alpha$-and $\gamma$-Fol-PEG-DSPE), were produced by solid phase peptide synthesis. Three types of doxorubicin (DOX)-loaded polymeric micelles (MPEG-DSPE-DOX and $\alpha$ - $/ \gamma$-Fol-PEG-DSPEDOX micelles) were prepared via the film formation method. Compared with MPEG-DSPE-DOX micelles, the $\alpha$ - / $\gamma$-Fol-PEG-DSPE-DOX micelles presented a higher cellular uptake behavior in the live cell study. Cell viability percentages were $81.8 \%, 57.3 \%, 56.6 \%$ at 2 hours for MPEG-DSPE-DOX, $\alpha$ - and $\gamma$-Fol-PEG-DSPEDOX micelles, respectively $(p<0.05)$. Using the KB xenograft tumor model, both $\alpha$ - and $\gamma$-folate-conjugated micelles were found to have better antitumor effects with lower toxicity in comparison with MPEG-DSPE-DOX micelles. No difference in in vivo antitumor efficacy was found between $\alpha$-and $\gamma$-Fol-PEG-DSPE-DOX micelles. The folate-conjugated micelles might be a potentially useful strategy for tumor targeting of therapeutic agents, whether grafting with folic acid through $\alpha$ - or $\gamma$-carboxyl groups.
\end{abstract}

Keywords: Isomeric $\alpha-/ \gamma$-folate conjugation - polymeric micelles - tumor targeting

Asian Pac J Cancer Prev, 15 (17), 7363-7369

\section{Introduction}

Folate receptors (FRs), which are GPI-anchored membrane protein involved in folate endocytosis, are often over-expressed on tumor cells including malignancies of the ovary, brain, kidney, breast, myeloid cells and lungs (Elnakat and Ratnam, 2004; Chen et al., 2013). Although a few normal tissues have also been found expresses folate receptors, these receptors located on the apical membrane of polarized epithelia are not accessible to blood-borne folate-derived conjugates. With high affinity towards the FRs, as a targeting ligand, folic acid has various advantages, such as low molecular weight and stability. In order to construct a folic acid-modified drug delivery system, folic acid is often covalently conjugated with PEG through its $\alpha$ - or $\gamma$-carboxyl group to connect with drug delivery system. Nevertheless, the binding ability for $\alpha$ and $\gamma$-folate regioisomers to FRs was disputed. Wang and colleagues showed that the cell surface FRs recognizes folate derivatives at the $\gamma$ - but not $\alpha$-carboxylate (Wang et al., 1996; Wang et al., 1997). In contrast, it was recently reported that both $\alpha$ - and $\gamma$-folate derivatives are able to bind to folate receptor-positive cells at virtually identical levels (Leamon et al., 1999; Bettio et al., 2006; Zong et al., 2012).
Doxorubicin (DOX) is widely used antitumor drug in the treatment of many types of cancers; however, it exhibits serious cardiac toxicity (Ashrafi and Roshan, 2012). In order to reduce side effect of antitumor agents, various drug delivery systems have been developed, such as polymer conjugates, polymeric micelles and liposomes (Torchilin, 2004; Chang et al., 2009; Khemapech et al., 2013; Xu et al., 2013). As an important member of drug delivery systems, polymeric micelles have attracted significant attention in tumor-targeted drug delivery systems recently. Polymeric micelles formed from amphiphilic copolymers are found to have high stability, controllable drug release profiles, long systemic circulation time and increased accumulation in tumor tissues via EPR effects (Torchilin, 2007; Wang et al., 2010). Amphiphilic copolymers containing hydrophobic block and hydrophilic block play a critically essential role in the form of polymeric micelles. Even though there are many options for the hydrophobic block, for the hydrophilic segment, polyethylene glycol (PEG) is the most frequently used one. Micelles coated with PEG have the ability to escape from the reticuloendothelial system and circulate in the blood for long periods of time (Hawley et al., 1995; VertutDoi et al., 1996; Gabizon et al., 2004). As a hydrophobic segment, distearoylphosphatidylethanol-amine (DSPE) represents

${ }^{1}$ Key Laboratory of Smart Drug Delivery (Fudan University), Ministry of Education, Department of Pharmaceutics, ${ }^{2}$ Department of Medicinal Chemistry, School of Pharmacy, Fudan University, Shanghai, China ${ }^{\circledR}$ Equal contributors *For correspondence: liumin@shmu.edu.cn 
good biocompatibility. When this hydrophobic DSPE conjugates to PEG, PEG-DSPE micelles are formed, which have suitable nanoscale $(-20 \mathrm{~nm})$, high stability, and low toxicity (Lukyanov et al., 2002; Torchilin, 2002).

Due to the study on the difference in the ability to bind to FRs between $\alpha$ - and $\gamma$-folate regioisomers, furthermore, the affinity of the two folate regioisomers for FRs was controversial, it cannot be assumed that the necessity of preparing the pure $\gamma$-folate regioisomers. In order to figure out the perplexity of the folate regioisomers, we planned to apply DOX as model drug and polymeric micelles as drug delivery system to study the folate derivatives. We adopted solid phase peptide synthesis to synthesize two isomeric copolymers, $\alpha$ - and $\gamma$-Fol-PEG-DSPE. Then we prepared both $\alpha$ - and $\gamma$-Fol-PEG-DSPE-DOX micelles to investigate their antitumor efficacy in vitro and in vivo.

\section{Materials and Methods}

Folic acid was purchased from Shisheng Biotechnology Co., LTD (Shanghai, China). Maleimide-poly (ethyleneglycol)-distearoylphosphatidylethanolamine (Mal-PEG-DSPE, MW3400) was obtained from JenKem technology Co., LTD (Beijing, China). Methoxy-poly (ethyleneglycol) -distearoylphosphatidylethanolamine (MPEG-DSPE, MW 2000) was purchased from Lipoid (German). Fomc-Cys (Trt) -2-Chlorotritylchloride resin was obtained from Langene Bioscience Co., LTD (Xi'an, China). 1-hydroxbenzotriazole (HOBt) was purchased from Gil Bio (Shanghai, China). Benzotriazole-1-yloxytripyrrolidinophosphonium hexafluoro-phosphate (PyBOP) was obtained from GL Biochem LTD (Shanghai, China). HaiZheng Corp (Taizhou, China) and Biotum Corp (USA) provided doxorubicin Hydrochloride and fluorescent dye DiR, respectively. All other chemicals and components for buffer solutions were HPLC grade preparations. The human oropharyngeal epidermoid carcinoma cell line (KB) was kindly provided by Shanghai Cancer Institute. RPMI 1640 medium, folate-free RPMI 1640 medium, Tripsin-EDTA, Fetal bovine serum (FBS) and pen strep $(+10000$ uints/ml penicillin and +10000 $\mu \mathrm{g} / \mathrm{mL}$ streptomycin) were purchased from Gibco Co (USA). Nude mice (male) were provided by Shanghai Slac Laboratory animal. Handling and care of animals were performed with the approval of Institutional Authority for Laboratory Animal Care.

\section{Synthesis of $\alpha$-Fol-Cys and $\gamma$-Fol-Cys}

Two Isomeric Fol-Cys conjugates were synthesized through the solid phase peptide synthesis method. The commercially available 2-chlorotritylchloride resin was coupled to Fomc-Cys (Trt)-OH. Resin (1.17 g) was swelled with dichlormethane (DCM) and washed with $\mathrm{N}, \mathrm{N}$-Dimethylformamide (DMF) three times, and the resin-bound Fomc-Cys was successively treated with $25 \%$ piperidine in DMF. Next, keeping all the operations in the dark, $0.7 \mathrm{~g}$ PyBop and $0.18 \mathrm{~g} \mathrm{HOBt}$ were added into 20 $\mathrm{ml}$ of DMF, dissolving $0.6 \mathrm{mg}$ of folic acid, and stirred for $5 \mathrm{~min}$, and added the solution into the resin mixture to react for 24 hours. Then, added trifluoroacetic acid (TFA) to the reaction solution and placed it in an ice bath for 1.5 hours. After that, the filtrate was collected by vacuum filtration under pressure. Then cold anhydrous diethyl ether was added into the filtrate to obtain a precipitate, which represented crude Fol-Cys. In order to obtain pure $\alpha$-Fol-Cys and $\gamma$-Fol-Cys, the crude Fol-Cys was purified by preparative HPLC (Waters, $600 \mathrm{E}$, USA) using a Waters System PrepTM C18 reversed phase column ( $7 \mu \mathrm{m}, 19 \times 30$ mm, Eluent A, water with $0.1 \%$ TFA; Eluent B, acetonitrile with $0.1 \%$ TFA; gradient, $5 \mathrm{~B}$ to $25 \% \mathrm{~B}$ over 30 minutes; flow rate, 10 mL/min; UV@280 nm).

\section{Synthesis of $\alpha$ - and $\gamma$-Fol-PEG-DSPE}

Mal-PEG-DSPE was dissolved in a solution of DMF. Then excessive $\alpha$-Fol-Cys was added into the solution and stirred at room temperature in the dark. The reaction was monitored by HPLC analysis (Agilent 1100 series, USA). Excess $\alpha$-Fol-Cys was removed by preparative HPLC (Sepax C4 10×250 mm $5 \mu \mathrm{m}$, Eluent A, water with $0.1 \%$ TFA; Eluent B, acetonitrile with $0.1 \%$ TFA; gradient, 70 B to $90 \%$ B over 30 minutes; flow rate, $3 \mathrm{~mL} / \mathrm{min}$; UV @ $220 \mathrm{~nm}$ ), and the purity of the $\alpha$-Fol-PEG-DSPE obtained was confirmed by NMR analysis. $\gamma$-Fol-PEG-DSPE was prepared and purified using the same methodologies as described above.

\section{Preparationand Characterization of Micelles}

Micelles were successfully prepared using the film formation method. Briefly, 0.6 mg Doxorubicin hydrochloride was stirred with 2 molar ratio of triethylamine in methanol to remove the hydrochlorate salt and then mixed with $5 \mathrm{mg}$ MPEG-DSPE and $\alpha$-or $\gamma$-FolPEG-DSPE/MPEG-DSPE (molar ratio = 100:1) dissolved in chloroform and dried in a rotary evaporation to form a thin film at $37^{\circ} \mathrm{C}$, which was re-suspended in $10 \mathrm{mM}$ HEPES-buffered saline ( $\mathrm{pH}$ 7.4) at room temperature. To remove un-incorporated doxorubicin, eluting with $10 \mathrm{mM}$ HEPES-buffered saline, the micelles were passed through a gel column filled with Sephadex G-50. The solution was determined by high-performance liquid chromatography (HPLC) show that the final concentration of doxorubicin in micelles was $1 \mathrm{mg} / \mathrm{mL}$ Micelle size was measured by dynamic light scattering (DLS) using NICOMP 380 ZLS Zeta-potential/Particle System (Malvern Zetasizer Nano Series, Britain). The morphological examination of micelles was performed using a transmission electron microscope (TEM, Joel JEM-1230, Japan). Fluorescent dye DiR and Rodamine B loaded micelles were prepared by dissolving fluorescein in acetonitrile and the later steps were the same as the preparation of DOX-loaded micelles.

\section{In Vitro Antitumor Efficacy of Micelles}

$\mathrm{KB}$ cells $\left(4 \times 10^{3}\right.$ cells $\left./ \mathrm{ml}\right)$ were seeded onto a $96-$ well plate in $200 \mu \mathrm{l}$ of RPMI 1640 medium containing $10 \%$ FBS.After 24 hours, the medium was replaced with folatefree RPMI 1640 medium. DOX-loaded micelles (DOX concentration $3.45 \mu \mathrm{mol} / \mathrm{L}$, equivalently) in folate-free RPMI1640 medium were added to the 96-well plate for 2 hours or 4 hours at $37^{\circ} \mathrm{C}$. The cells were subsequently incubated in fresh growth media for a total of 48 hours 
before cell viability was assessed in each group. The viability of cells was measured using the tetrazolium dye method (MTT assay).

\section{Uptake of macrophages}

Flow cytometry was used to compare the intracellular uptake of Rhodamine B-loaded micelles by Macrophages cell. RAW 264.7 cells were seeded in 6-well plates (each well 105 cells) and grown for 1 day in DMEM medium containing $10 \%$ FBS. The cells were incubated for 30 minutes at $37^{\circ} \mathrm{C}$ with free Rhodamine $\mathrm{B}$ or Rhodamine B-loaded micelles. After the medium was removed, RAW 264.7 cells was washed with PBS, detached by $0.25 \%$ Trypsin-EDTA, fixed by $1 \%$ formaldehyde in PBS, resuspended in $1 \mathrm{ml}$ of $\mathrm{PBS}$, and immediately analyzed by flow cytometry.

\section{In vivo optical imaging of micelle biodistribution}

In vivo, biodistribution tests were carried out with DiR-loaded micelles using optical imaging. KB cells were transplanted into the shoulder of male nude mice by subcutaneous injection of $1 \times 10^{6}$ cells suspended in cell culture media. When the size of the tumor had reached a size of approximately $300 \mathrm{~mm}^{3}$, DiR loaded MPEG-DSPE, $\alpha$-and $\gamma$-Fol-PEG-DSPE micelles were intravenously injected into KB tumor-bearing nude mice through the tail vein. $2 \mathrm{~h}$ and $8 \mathrm{~h}$ after injection, the mice were transferred to a Maestro In-Vivo Imaging System for observation.

\section{In vivo antitumor activity}

The same manner as described above was used to establish the KB tumor-bearing nude mice model. The mice were kept on a folate-deficient diet exclusively throughout the study. Two weeks after the tumor transplanted, the mice were randomly assigned to five groups $(n=6)$ and were treated five times at 2-day intervals with free DOX, MPEG-DSPE-DOX micelles, $\alpha$ - and $\gamma$-Fol-PEG-DSPE-DOX micelles (2 $\mathrm{mg} / \mathrm{kg}$ per dose), and $200 \mu \mathrm{l}$ of physiological saline (blank control) via tail vein injection. Tumor size was determined using serial calipers (GuangLu ${ }^{\circledR}$, China) and measurements were made every two days to calculate the volumes using the formula length $\times$ (width) $2 / 2$. The inhibition rate of tumor growth $(\mathrm{IR} \%):=(\mathrm{Vc}-\mathrm{Vt}) / \mathrm{Vc} \times 100 \%$, where $\mathrm{Vc}$ and $\mathrm{Vt}$ are the mean tumor size of the blank control and treated mice respectively. $\mathrm{Vt} / \mathrm{Wt}$ stands for the relationship between tumor size and mice weight, where $\mathrm{Wt}$ is the mice weight.

\section{Histopathological Analysis}

After the study of in vivo antitumor activity, all of the surviving nude mice were sacrificed and dissected. The hearts of mice were fixed in $10 \%$ neutral buffered formalin and embedded in paraffin to make tissue slice. All the groups of mice' heart tissue slice were stained with haematosylin and eosin. Then the stained sections were observed under microscope and photographed.

\section{Statistical analysis}

Differences of the vivo antitumor effect between test groups were assessed using an Unpaired Student's t-Test using the significance level of $p<0.05$.

\section{Results}

Characterization of $\alpha$-Fol-Cys and $\gamma$-Fol-Cys

The isomeric Fol-Cys conjugates were synthesized using the Fmoc-protected solid phase peptide synthesis method. We separated the crude Fol-Cys into $\alpha$-Fol-Cys and $\gamma$-Fol-Cys by preparative HPLC. The purified $\alpha$-and $\gamma$-Fol-Cys were distinguished by their retain times which were 21.30 and $19.21 \mathrm{~min}$ respectively, using ananalytical HPLC method (Agilent 1100 series). The data of 1H-NMR (400 MHz, DMSO-d6) analyses were as follow: $\delta$ (ppm) for folic acid: $8.62\left(\mathrm{~s}, 1 \mathrm{H}, \mathrm{C}_{7}-\mathrm{H}\right), 7.63(\mathrm{~d}, \mathrm{~J}=8.4 \mathrm{~Hz}, 2 \mathrm{H}$, b), $6.61(\mathrm{~d}, \mathrm{~J}=8.4 \mathrm{~Hz}, 2 \mathrm{H}, \mathrm{a}), 4.46\left(\mathrm{~d}, \mathrm{~J}=5.9 \mathrm{~Hz},-\mathrm{CH}_{2}\right.$, $2 \mathrm{H}), 4.32(\mathrm{~m}, 1 \mathrm{H}, 4-\mathrm{CH}), 2.31\left(\mathrm{~m}, 2 \mathrm{H}, 2-\mathrm{CH}_{2}\right), 1.85-2.07$ $\left(\mathrm{m} \times 2,2 \mathrm{H}, 3-\mathrm{CH}_{2}\right), 6.93(\mathrm{t}, \mathrm{J}=5.9 \mathrm{~Hz}, 1 \mathrm{H},-\mathrm{NH}), 6.91$ (b, 2H, - $\left.\mathrm{NH}_{2}\right), 8.12(\mathrm{~m}, 1 \mathrm{H},-\mathrm{NH}$ of Glu), 12.31 (b, 2H, $-\mathrm{COOH} \times 2), 11.40(\mathrm{~s}, 1 \mathrm{H},-\mathrm{OH}) . \delta(\mathrm{ppm})$ for $\alpha$-Fol-Cys: $8.62\left(\mathrm{~s}, 1 \mathrm{H}, \mathrm{C}_{7}-\mathrm{H}\right), 7.63(\mathrm{~d}, \mathrm{~J}=8.4 \mathrm{~Hz}, 2 \mathrm{H}, \mathrm{b}), 6.61$ (d, J=8.4 $\mathrm{Hz}, 2 \mathrm{H}, \mathrm{a}), 4.46$ (d, J=5.9 Hz, $\left.-\mathrm{CH}_{2}, 2 \mathrm{H}\right), 4.26(\mathrm{~m}, 1 \mathrm{H}$, 4-CH), $2.29\left(\mathrm{~m}, 2 \mathrm{H}, 2-\mathrm{CH}_{2}\right), 1.72-2.00\left(\mathrm{~m} \times 2,2 \mathrm{H}, 3-\mathrm{CH}_{2}\right)$, $6.93(\mathrm{t}, \mathrm{J}=5.9 \mathrm{~Hz}, 1 \mathrm{H},-\mathrm{NH}), 6.91\left(\mathrm{~b}, 2 \mathrm{H},-\mathrm{NH}_{2}\right), 8.12(\mathrm{~m}$, $1 \mathrm{H},-\mathrm{NH}$ of Glu $), 12.34$ (b, $2 \mathrm{H},-\mathrm{COOH} \times 2), 11.40(\mathrm{~s}, 1 \mathrm{H}$, $-\mathrm{OH}), 2.64-2.79$ (m, 2H, $-\mathrm{CH}_{2}$ of $\left.\mathrm{Cys}\right), 4.34$ (m, 1H, -CH of Cys), 7.18 ( $\mathrm{m}, 1 \mathrm{H}, \mathrm{NH}$ of Cys). $\delta$ (ppm) for $\gamma$-Fol-Cys: 8.62 (s, 1H, C $-\mathrm{H}), 7.63$ (d, J=8.4 Hz, 2H, b), 6.61 (d, J $=8.4 \mathrm{~Hz}, 2 \mathrm{H}, \mathrm{a}), 4.46\left(\mathrm{~d}, \mathrm{~J}=5.9 \mathrm{~Hz},-\mathrm{CH}_{2}, 2 \mathrm{H}\right), 4.28(\mathrm{~m}$, $1 \mathrm{H}, 4-\mathrm{CH}), 2.24\left(\mathrm{~m}, 2 \mathrm{H}, 2-\mathrm{CH}_{2}\right), 1.85-2.07(\mathrm{~m} \times 2,2 \mathrm{H}$, $\left.3-\mathrm{CH}_{2}\right), 6.93(\mathrm{t}, \mathrm{J}=5.9 \mathrm{~Hz}, 1 \mathrm{H},-\mathrm{NH}), 6.91\left(\mathrm{~b}, 2 \mathrm{H},-\mathrm{NH}_{2}\right)$, $8.12(\mathrm{~m}, 1 \mathrm{H},-\mathrm{NH}$ of Glu $), 12.34$ (b, $2 \mathrm{H},-\mathrm{COOH} \times 2), 11.40$ (s, $1 \mathrm{H},-\mathrm{OH}), 2.64-2.79\left(\mathrm{~m}, 2 \mathrm{H},-\mathrm{CH}_{2}\right.$ of $\left.\mathrm{Cys}\right), 4.34(\mathrm{~m}$, 1H, -CH of Cys), 7.18 (m, 1H, NH of Cys). The 1H-NMR data showed a stronger chemical shift of 4-CH (link to the $\alpha$-carboxyl group) of folic acid, which indicated that Cys had reacted with the $\alpha$-carboxyl group of folic acid. While the stronger chemical shift of the 2-CH2 (link to the $\gamma$-carboxyl group) of folic acid demonstrated that Cys had reacted with the $\gamma$-carboxyl group of folic acid. ESI (+) MS calculated for $\mathrm{C}_{22} \mathrm{H}_{24} \mathrm{H}_{8} \mathrm{O}_{7} \mathrm{~S}(\mathrm{M}+\mathrm{H}) 544.2$, observed 545.3 for two isomeric Fol-Cys compounds.

\section{Characterization of $\alpha$ - and $\gamma$-Fol-PEG-DSPE}

The purity of $\alpha$ - and $\gamma$-Fol-PEG-DSPE was confirmed by HPLC. The retention time of $\alpha$-Fol-PEG-DSPE, $\gamma$-FolPEG-DSPE and Mal-PEG-DSPE was 14.11, 14.08 and 11.2 min, respectively. A single peak of $\alpha$-Fol-PEG-DSPE $(\gamma$-Fol-PEG-DSPE) without Mal-PEG-DSPE and $\alpha$-FolCys ( $\gamma$-Fol-Cys) was obtained. $\delta$ (ppm) for Fol-PEGDSPE as determined by ${ }^{1} \mathrm{H}-\mathrm{NMR}(400 \mathrm{MHz}, \mathrm{DMSO}-\mathrm{d} 6)$ was: 8.62 (s, 1H, $\left.\mathrm{C}_{7}-\mathrm{H}\right), 7.63$ (d, J=8.4 Hz, 2H, b), 6.61 (d, J=8.4 Hz, 2H, a), 4.46 (d, J=5.9 Hz, $\left.-\mathrm{CH}_{2}, 2 \mathrm{H}\right), 4.28$ (m, 1H, 4-CH), 2.24 (m, 2H, 2- $\left.\mathrm{CH}_{2}\right), 1.85-2.07(\mathrm{~m} \times 2,2 \mathrm{H}$, $\left.3-\mathrm{CH}_{2}\right), 6.93(\mathrm{t}, \mathrm{J}=5.9 \mathrm{~Hz}, 1 \mathrm{H},-\mathrm{NH}), 6.91\left(\mathrm{~b}, 2 \mathrm{H},-\mathrm{NH}_{2}\right)$, $8.12(\mathrm{~m}, 1 \mathrm{H},-\mathrm{NH}$ of Glu $), 12.34$ (b, 2H, $-\mathrm{COOH} \times 2), 11.40$ (s, $1 \mathrm{H},-\mathrm{OH}) ; 3.1-3.58\left(\mathrm{~m},-\mathrm{OCH}_{2} \mathrm{CH}_{2}\right)$ for PEG and 0.81 $\left(\mathrm{s}, 6 \mathrm{H},-\mathrm{CH}_{3}\right), 1.19\left(\mathrm{~s}, 64 \mathrm{H},-\mathrm{CH}_{2}\right)$ for DSPE.

\section{Characterization of Micelles}

The particle size and polydispersity index (PDI) of DOX-loaded micelles were $20 \mathrm{~nm} \pm 5 \mathrm{~nm}$ and 0.1 respectively. The morphology of the micelles was investigated by TEM (Figure 1). It could be seen that the 

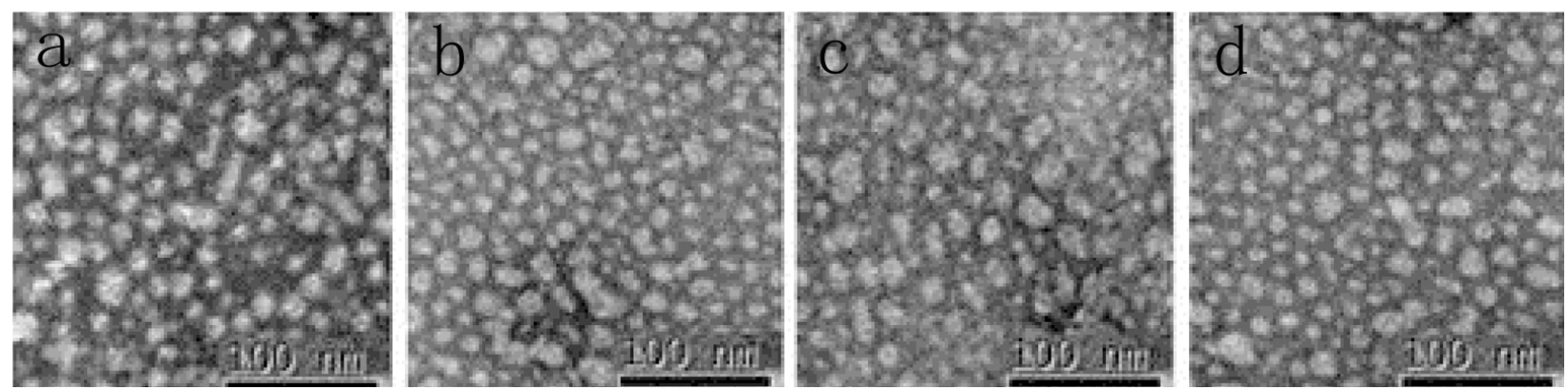

Figure 1. Transmission Electron Microscope Photographs of Micelles; (a) MPEG-DSPE-DOX micelles; (b) $\alpha$-FolPEG-DSPE-DOX micelles; (c) $\gamma$-Fol-PEG-DSPE-DOX micelles; (d) MPEG-DSPE-DOX micelles after storage at $4{ }^{\circ} \mathrm{C}$ for 4 months

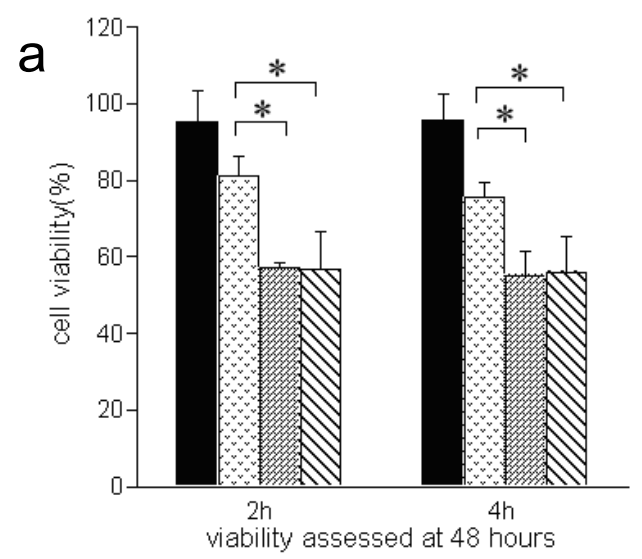

MPEG-DSPE micelles

MPEG-DSPE-DOX micelles

$\alpha$-Fol-PEG-DSPE-DOX micelles

तV $\mathrm{y}$-Fol-PEG-DSPE-DOX micelles

b
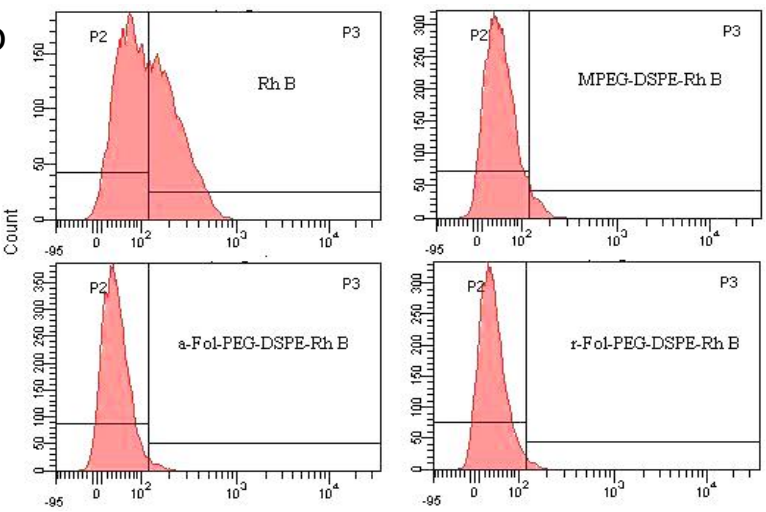

Figure 2. (a)The MTT assay was used to determine the differential cytotoxicity of MEPG-DSPE micelles without DOX, MPEG-DSPE-DOX, $\alpha$ - and $\gamma$-Fol-PEG-DSPEDOX micelles, following incubated of KB cells for 2 hours (left) or 4 hours (right); (* $\left.{ }^{*}<0.05\right)$ (b) The evaluation of macrophages RAW264.7 cells were analyzed through flow cytometry, incubated with free Rhodamine B and three kinds of Rhodamine B-loaded micelles

self-assembled micelles were well dispersed as individual nanoparticles with regular spherical shape and no drug crystal was visible, which confirmed that the micellization process did occur. We stored the micelles solution at $4^{\circ} \mathrm{C}$ for 4 months and then made it passed through a gel column (Sephadex G-50). That no drug dissociation was determined proved that DOX-loaded micelles which diluted in HEPES-buffered saline ( $\mathrm{pH} 7.4$ ) are sufficiently stable at $4^{\circ} \mathrm{C}$ in 4 months. The encapsulation and drug loading efficiency of DOX incorporated into micelles were $85.2 \% \pm 0.6 \%$ and $10 \% \pm 1.2 \%$, respectively. The $\alpha$ - and $\gamma$-Fol-PEG-DSPE-DOX micelles showed no difference in size, encapsulation efficiency and drug loading efficiency when compared with MPEG-DSPE-DOX micelles.

\section{In Vitro Antitumor Efficacy}

The antitumor efficacy of DOX-loaded micelles on KB cells was analyzed through the MTT assay. Our data suggests that $\alpha-\gamma$-folate-conjugated micelles are significantly more cytotoxic as compared with the MPEGDSPE-DOX micelles. The cell viability of MPEG-DSPEDOX, $\alpha$ - and $\gamma$-Fol-PEG-DSPE-DOX micelles were $81.8 \%, 57.3 \%, 56.6 \%$ at $2 \mathrm{~h}$ and $75.7 \%, 55.2 \%, 56.1 \%$ at $4 \mathrm{~h}$ respectively $(p<0.05)$ (Figure $2 \mathrm{a})$.

\section{Uptake of macrophages}

In order to evaluate the influences of PEG-DSPE micelles on macrophage phagocytosis, RAW 264.7 cells were incubated with Rhodamine B-loaded micelles for $30 \mathrm{~min}$, and the uptake was monitored by flow cytometry. For the free Rhodamine B, 36.6 percent of macrophages phagocytized the Rhodamine $\mathrm{B}$, nevertheless, when Rhodamine B was encapsulated in micelles, the fluorescent uptake of macrophages decreased significantly. The percentages of RAW 264.7 cells phagocytizing the Rhodamine B are $4.40 \%, 1.51 \%$ and $1.80 \%$, which were incubated with MPEG-DSPE-RhB micelles, $\alpha$-Fol-PEGDSPE-RhB micelles and $\gamma$-Fol-PEG-DSPE-RhB micelles respectively. As expected, the PEG-DSPE micelles could inhibit the uptake of Rbodamine B by RAW 264.7 cells, in comparison with free Rhodamine B, which was inhibited up to $94.2 \%$ (Figure 2b). These results demonstrated that no matter the $\alpha$-Fol-PEG-DSPE-RhB micelles or the $\gamma$-Fol-PEG-DSPE-RhB could avoid macrophage phagocytosis to prolong the circulation time.

\section{In Vivo Optical Imaging of Micelle Biodistribution}

After DiR loaded micelles were injected, through near infrared spectroscopy, we observed the distribution of micelles in KB tumor-bearing nude mice. The fluorescence of micelles was found to accumulate mainly in tumors at $2 \mathrm{~h}$ and $8 \mathrm{~h}$ in vivo (Figure 3 ). The tumors could be delineated from the surrounding normal tissue as early as $2 \mathrm{~h}$ after injection. Compared with MPEG-DSPE-DOX micelles, the $\alpha$-and $\gamma$-Fol-PEG-DSPE micelles showed a stronger intensity at all tested time points. 

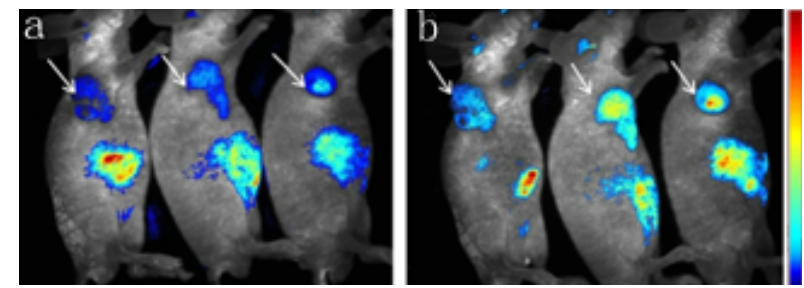

Figure 3. Optical Imaging Result for Tumor Bearing Nude Mice taken using DiR loading Micelles in at Times Periods (a: $2 \mathbf{h}$; b: 8 h). Tumors are highlighted by the white arrows (from left to right, MPEG-DSPE, $\alpha$ - and $\gamma$-Fol-PEG-DSPE micelles in order)

\section{In Vivo Antitumor Activity}

In the KB tumor-bearing mice model, free DOX and DOX-loaded micelles exhibited the different in vivo antitumor efficiency. The tumor volume growth (Figure 4a) and the changes in the body weight of mice (Figure 4b) were examined over a 26-dayperiod. It can be seen that the tumor growth in mice treated with $\alpha$ - or $\gamma$-Fol-PEGDSPE-DOX micelles was suppressed more strongly than those treated with DOX and MPEG-DSPE-DOX micelles and was considered as significant $(p<0.05)$. After 26 days, the IRs $(\%)$ were $50.4 \%, 42 \%, 69.6 \%$ and $74.8 \%$ for free DOX, MPEG-DSPE-DOX micelles, $\alpha$ - and $\gamma$-Fol-PEGDSPE-DOX micelles respectively. Indeed, the $\alpha$ - and $\gamma$-folate-modified micelles suppressed the tumor growth more than that of MPEG-DSPE-DOX micelles at this stage. Moreover, no significant difference in the size of tumors was observed between $\alpha$ - and $\gamma$-Fol-PEG-DSPEDOX micelle treated animals.

Although free DOX and all kinds of DOX-loaded micelles possessed antitumor activity, a sharp decrease in body weight during the treatment was observed in the free DOX group. The results suggested serious toxicity levels of free DOX at the given dose compared with DOX-loaded micelles. Additionally, empty micelles showed no signs of toxicity (data not shown). The ratio of tumor volume to mice weight was calculated and shows that the $\mathrm{Vt} / \mathrm{Wt}$ of free DOX and MPEG-DSPE-DOX micelles was higher than $\alpha$ - and $\gamma$-Fol-PEG-DSPE-DOX micelles (Figure 4c). That means the $\alpha$ - and $\gamma$-Fol-PEG-DSPE-DOX micelles have a better antitumor effect and lower toxicity as compared to free DOX and MPEG-DSPE-DOX micelles.

\section{Histopathological Analysis}

In order to observe the doxorubicin-induced cardiac toxicity in mice, the hearts of mice were prepared for histological analysis. The control group showed regular myofiber, maintained sarcotubular structure and abundant blood vessel among myocardium. Whereas myocardial fibers necrosis and myocardial damage were observed in the group treated with free doxorubicin. There is little evidence of morphological damage were seen in the three groups: MPEG-DSPE-DOX micelles group, $\alpha$-Fol-PEG-DSPE-DOX micelles group and $\gamma$-Fol-PEGDSPE-DOX micelles group (Figure 5). The comparisons between groups demonstrate that polymeric micelles encapsulating doxorubicin could lower the cardiac toxicity of doxorubicin.
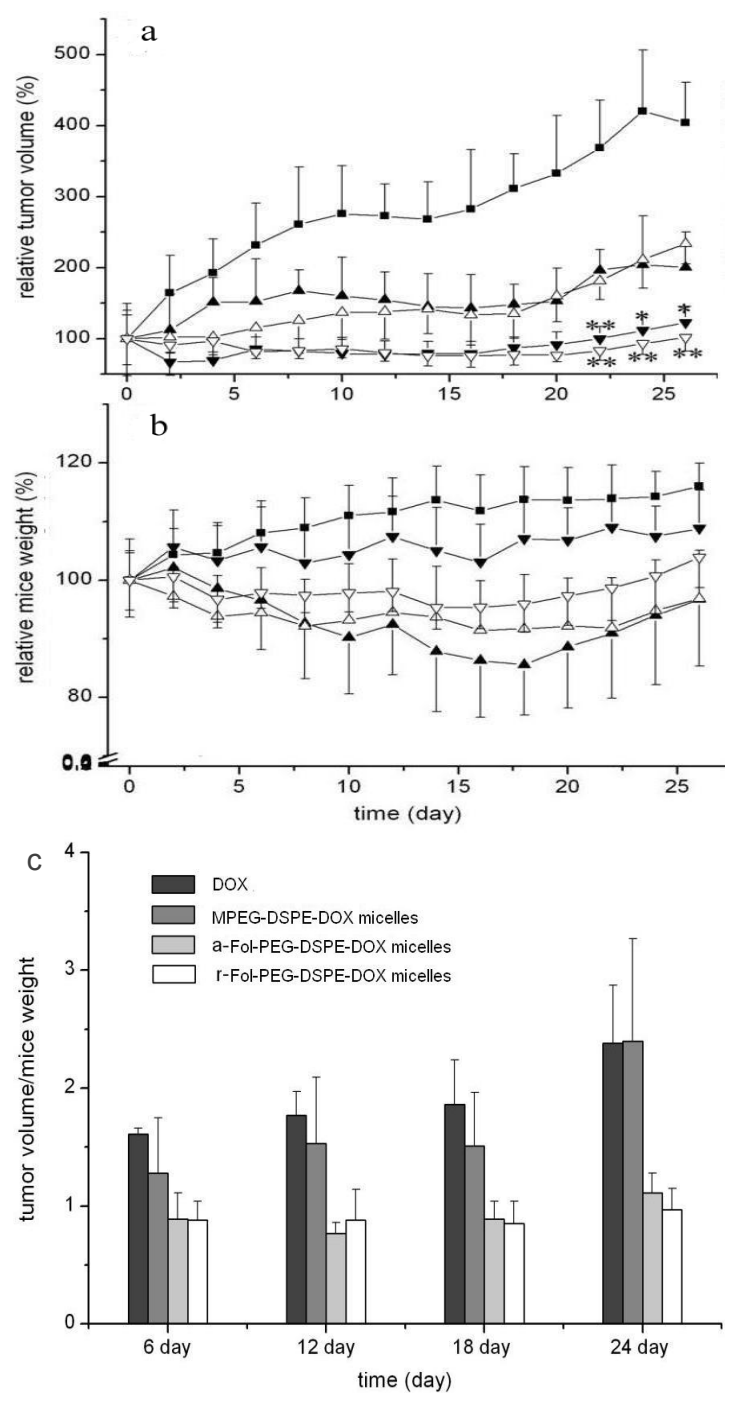

Figure 4. The Experiment of Tumor Volume Growth (a) and body weight change (b) in nude mice bearing $\mathrm{KB}$ cell xenografts $(\mathbf{n}=\mathbf{6})$. The mice were randomly assigned to five group and were treated five times (days 0,3,6, 9, $12)$ at a 2-day interval $(2 \mathrm{mg} / \mathrm{kg}$ per dose, $10 \mathrm{mg} / \mathrm{kg}$ total of DOX). $(* * p<0.01, * p<0.05$, vs MPEG-DSPE-DOX micelles group). $(-\mathbf{-}-$ : control, $-\mathbf{\Delta}-$ : DOX, $-\triangle-$ : MPEG-DSPE-DOX micelles, $-\boldsymbol{\nabla}-: \alpha$-Fol-PEGDSPE-DOX micelles, $-\nabla-: \gamma$-Fol- PEG-DSPE-DOX micelles). (c) The ratio of tumor volume and mouse body weight at $6,12,18$, and 24 days

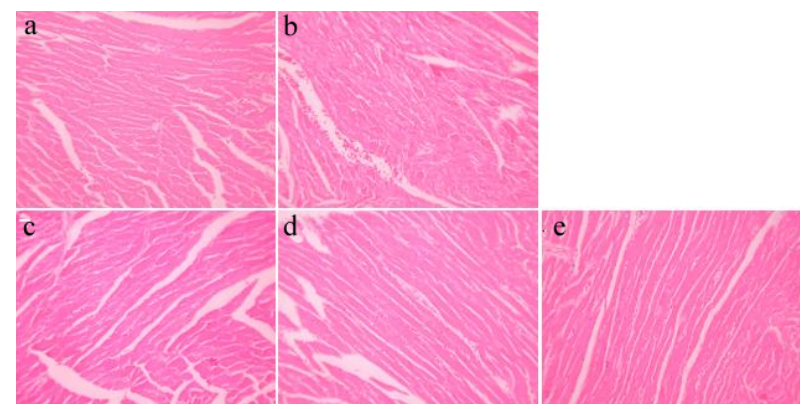

Figure 5. The photomicrograph of heart with mice (shown at $\times 100$ magnification and stained with HE); (a) control; (b) free DOX; (c) MPEG-DSPE-DOX micelles; (d) $\alpha$-Fol-PEG-DSPE-DOX micelles; (e) $\gamma$-Fol-PEGDSPE-DOX micelles 


\section{Discussion}

A number of studies have shown that folate receptors are over expressed in malignant cells in comparison to that of normal tissues. This differential expression has lead to the development of folate receptor-mediated diagnostic and therapeutic agents for the treatment of various cancers (Liu et al., 2005; Park et al., 2005). In this study, we have prepared and evaluated folate-conjugated micelles as a potential antitumor drug delivery system.

In this study, folic acid was conjugated with MalPEG-DSPE to obtain the targeting material, Fol-PEGDSPE. Maleimide, presenting within Mal-PEG-DSPE, can react with hydrosulfide groups with high specificity and was used in the preparation and modification of the reported folic acid cysteine (Cys) complexes. To obtain the complexes, the amino group of Cys needs to react with $\alpha$ - or $\gamma$-carboxyl groups of folic acid; however, the reaction without selectivity. We envisaged that the reaction would lead to the production of three products, including $\alpha$-Fol-Cys, $\gamma$-Fol-Cys and Cys-Fol-Cys. Considering that $\alpha$-Fol-Cys, $\gamma$-Fol-Cys and Cys-Fol-Cys have a different chromatography behavior on $\mathrm{C} 18$ columns, we adopted a preparative HPLC method to obtain highly pure samples of both $\alpha$-Fol-Cys and $\gamma$-Fol-Cys.

It has previously been reported that Fol-PEG-PLA, Fol-PEG-PCL micelles and Fol-PEG-DSPE may be taken up by tumor cells through a receptor-mediated endocytosis process. The above micelles showed higher cell toxicity than folate-unconjugated micelles (Yoo and Park, 2004; Han et al., 2009; Liu et al., 2014). In this study, the $\alpha$ - and $\gamma$-Fol-PEG-DSPE-DOX micelles were found to promote a greater cellular uptake of DOX as compared to the MPEG-DSPE-DOX micelles. We also designed a series of studies to determine micelles toxicity. Experiments were used in which cells were incubated for 2 hours or 4 hours, this allowed for the specific up take of each micelle through a folate receptor-mediated endocytosis process. Following this, an additional 48 hours incubation period measuring cell viability using the MTT assay was also conducted. The result showed that $\alpha$ - and $\gamma$-Fol-PEGDSPE-DOX micelles exhibit higher cytotoxicity than the MPEG-DSPE-DOX micelles.

In in vivo antitumor activity study, in nude mice bearing s.c. $\mathrm{KB}$ cells, tumor volume in the treated groups (both $\alpha$ - and $\gamma$-Fol-PEG-DSPE-DOX micelles) was smaller than that of free DOX and MPEG-DSPE-DOX micelles respectively. In addition, mice administered with DOX always showed loss of weight (Figure $4 b$ ). The $\alpha$ - and $\gamma$-Fol-PEG-DSPE-DOX micelles showed no significant effects upon the body weights of animals as compared with the control group. This finding indicates that the folate-conjugated micelles have reduced side effects. What's more, the Vt/Wt of free DOX and MPEGDSPE-DOX micelles was 2 times higher than that of the folate-conjugated micelles after 24 days (Figure 4c). That means the $\alpha$ - and $\gamma$-Fol-PEG-DSPE-DOX micelles had a greater antitumor effect and lower toxicity as compared with free DOX and MPEG-DSPE micelles. Through our study, the $\alpha$-Fol-PEG-DSPE-DOX micelles and $\gamma$-FolPEG-DSPE-DOX micelles were demonstrated to have the similar antitumor activity in vitro and in vivo. Given a support, the preparation of folate-conjuegated micelles will be simplified, due to there is no need to separate $\gamma$-folate-conjuegated micelles from the folate-conjuegated micelles. It maybe improves the operability and economics under the premise of efficacy assurance.

\section{Acknowledgements}

This work was supported by the National Science Foundation of China (Grant no. 30772655), the National Key Basic Research Program of China (Grant no. 2013CB932502), the National Science and Technology Major Project (Grant no. 2012ZX09304004). We are grateful to the reviewers for their helpful comments.

\section{References}

Bettio A, Honer M, Muller C, et al (2006). Synthesis and preclinical evaluation of a folic acid derivative labeled with F-18 for PET imaging of folate receptor-positive tumors. $J$ Nucl Med, 47, 1153-60.

Chang DK, Chiu CK, Kuo SY, et al (2009). Antiangiogenic targeting liposomes increase therapeutic efficacy for solid tumors. J Biol Chem, 284, 12905-16.

Chen C, Ke J, Zhou XE, et al (2013). Structural basis for molecular recognition of folic acid by folate receptors. Nature, 500, 486-9.

Elnakat H, Ratnam M (2004). Distribution, functionality and gene regulation of folate receptor isoforms: implications in targeted therapy. Adv Drug Deliv Rev, 56, 1067-84.

Gabizon A, Shmeeda H, Horowitz AT, et al (2004). Tumor cell targeting of liposome-entrapped drugs with phospholipidanchored folic acid-PEG conjugates. Adv Drug Deliv Rev, 56, 1177-92.

Han X, Liu J, Liu M, et al (2009). 9-NC-loaded folate-conjugated polymer micelles as tumor targeted drug delivery system: Preparation and evaluation in vitro. Int J Pharm, 372, 125-31.

Hawley AE, Davis SS, Illum L (1995). Targeting of colloids to lymph-nodes - influence of lymphatic physiology and colloidal characteristics. Advanced Drug Delivery Reviews, 17, 129-48.

Khemapech N, Oranratanaphan S, Termrungruanglert W, et al (2013). Salvage chemotherapy in recurrent platinumresistant or refractory epithelial ovarian cancer with carboplatin and distearoylphosphatidylcholine pegylated liposomal doxorubicin (Lipo-Dox (R)). Asian Pac J Cancer Prev, 14, 2131-5.

Leamon CP, De Prince RB, Hendren RW (1999). Folatemediated drug delivery: Effect of alternative conjugation chemistry. J Drug Targeting, 7, 157-69.

Liu B, Han SM, Tang XY, et al (2014). Cervical cancer gene therapy by gene loaded PEG-PLA nanomedicine. Asian Pac $J$ Cancer Prev, 15, 4915-8.

Liu M, Xu W, Xu LJ, et al (2005). Synthesis and biological evaluation of diethylenetriamine pentaacetic acidpolyethylene glycol-folate: a new folate-derived, $(99 \mathrm{~m}) \mathrm{Tc}$ based radiopharmaceutical. Bioconjuge Chem, 16, 1126-32.

Lukyanov AN, Gao ZG, Mazzola L, et al (2002). Polyethylene glycol-diacyllipid micelles demonstrate increased accumulation in subcutaneous tumors in mice. Pharm Res, 19, 1424-9.

Park EK, Kim SY, Lee SB, et al (2005). Folate-conjugated methoxy poly (ethylene glycol)/poly (epsilon-caprolactone) amphiphilic block copolymeric micelles for tumor-targeted drug delivery. J Control Release, 109, 158-68. 
Torchilin VP (2002). PEG-based micelles as carriers of contrast agents for different imaging modalities. Adv Drug Deliv Rev, 54, 235-52.

Torchilin VP (2004). Targeted polymeric micelles for delivery of poorly soluble drugs. Cell Mol Life Sci, 61, 2549-59.

Torchilin VP (2007). Micellar nanocarriers: Pharmaceutical perspectives. Pharm Res, 24, 1-16.

VertutDoi A, Ishiwata H, Miyajima K (1996). Binding and uptake of liposomes containing a poly (ethylene glycol) derivative of cholesterol (stealth liposomes) by the macrophage cell line J774: Influence of PEG content and its molecular weight. Bba-Biomembranes, 1278, 19-28.

Wang S, Lee RJ, Mathias CJ, et al (1996). Synthesis, purification, and tumor cell uptake of Ga-67-deferoxamine-folate, a potential radiopharmaceutical for tumor imaging. Bioconjugate Chem, 7, 56-62.

Wang S, Luo J, Lantrip DA, et al (1997). Design and synthesis of In-111 DTPA-folate for use as a tumor-targeted radiopharmaceutical. Bioconjugate Chemistry, 8, 673-9.

Wang YG, Wang RQ, Lu XY, et al (2010). Pegylated phospholipids-based self-assembly with water-soluble drugs. Pharm Res, 27, 361-70.

Xu X, Wang L, Xu HQ, et al (2013). Clinical comparison between paclitaxel liposome (Lipusu (r)) and paclitaxel for treatment of patients with metastatic gastric cancer. Asian Pac J Cancer Prev, 14, 2591-4.

Yoo HS, Park TG (2004). Folate receptor targeted biodegradable polymeric doxorubicin micelles. J Controll Release, 96, 273-83.

Zong H, Thomas TP, Lee KH, et al (2012). Bifunctional PAMAM dendrimer conjugates of folic acid and methotrexate with defined ratio. Biomacromolecules, 13, 982-91. 